\title{
Periventricular leukomalacia in very low birth weight preterm neonates with high risk for neonatal sepsis
}

\author{
Rita C. Silveira, ${ }^{1}$ Renato S. Procianoy, ${ }^{2}$ Juliana C. Dill, ${ }^{3}$ Cristine S. da Costa ${ }^{3}$
}

\begin{abstract}
Objective: To investigate the association between periventricular leukomalacia (PVL) and neonatal sepsis in very low birth weight infants (VLBWI).

Methods: We studied VLBWI with a clinical suspicion of infection who had been born at our institution between the 1st of August, 2005 and the 31st of July, 2007. Children were excluded if they died before reaching 14 days, had malformations of the central nervous system or congenital infections. Ultrasound brain scans were carried out on the third day and weekly up until the sixth week of life or discharge. Periventricular leukomalacia was diagnosed by persistent diffuse periventricular hyperechogenecity for more than 7 days, or by periventricular cysts. The VLBWI were separated into two groups on the basis of the presence or absence of PVL. Sepsis was defined as clinical manifestation plus a positive culture. The Mann-Whitney, chi-square and $t$ tests were applied followed by logistic regression.
\end{abstract}

Results: A total of 88 VLBWI were studied. Of these, 62 (70.5\%) survived and 51 (57.8\%) had PVL. Both groups were similar in terms of birth weight, gestational age, Apgar score, type of delivery, SNAPPE-II score, presence of necrotizing enterocolitis, persistent ductus arteriosus and deaths. Sepsis and mechanical ventilation were more common in the group with PVL ( 23.5 and $2.7 \%, p=0.005 ; 86$ and 59\%, $p=0.004$, respectively). Both of these were identified as, independent risk factors for PVL by logistic regression ( $p=0.027$ and 0.015 , respectively).

Conclusions: Chorioamnionitis has been defined as a risk factor for PVL. We have demonstrated that neonatal sepsis is also an important risk factor. We believe that the systemic inflammatory response is the principal factor involved in the etiopathogenesis of PVL among VLBWI.

J Pediatr (Rio J). 2008;84(3):211-216: Prematurity, neonatal sepsis, periventricular leukomalacia, very low birth weight.

\section{Introduction}

The increased survival of progressively more premature newborn infants has resulted in a significant number of preterm infants who grow up with neurocognitive abnormalities. $^{1,2}$ Currently, periventricular leukomalacia (PVL) is the principal neurological problem affecting children born extremely premature. Approximately $25 \%$ of newborn infants with birth weights below $1,500 \mathrm{~g}$ who survive to discharge exhibit moderate to severe permanent motor deficits, such as spastic diplegia. At school age, 25 to $50 \%$ of children who had been diagnosed with PVL manifest cognitive and learning deficits. ${ }^{3-5}$

The presentation of PVL is subclinical and so diagnosis is made by neuroimaging examinations (cerebral ultrasound and/or magnetic resonance). An early diagnosis of PVL can be achieved using transfontanelar cerebral ultrasound during the neonatal period used as screening for brain injuries. On the ultrasound brain scan, areas of periventricular hyperechogenecity can be observed which will later develop into periventricular cysts and/or diffuse hyperechogenic areas in the white

1. Doutora. Professora adjunta, Universidade Federal do Rio Grande do Sul (UFRGS), Porto Alegre, RS, Brazil.

2. Chefe, Serviço de Neonatologia, Hospital de Clínicas de Porto Alegre (HCPA), Porto Alegre, RS, Brazil. Professor titular, UFRGS, Porto Alegre, RS, Brazil.

3. Residente de $3^{\circ}$ ano (R3), Neonatologia.

Financial support: CNPQ-DECIT/MS and FIPE-HCPA.

No conflicts of interest declared concerning the publication of this article.

Suggested citation: Silveira RC, Procianoy RS, Dill JC, da Costa CS. Periventricular leukomalacia in very low birth weight preterm neonates with high risk for neonatal sepsis. J Pediatr (Rio J). 2008;84(3):211-216.

Manuscript received Nov 23 2007, accepted for publication Feb 142008.

doi:10.2223/JPED.1777 
matter. ${ }^{6,7}$ This diffuse component of PVL is common among extreme preterm newborns, and the focus appears to be associated with the inflammatory response resulting from ischemia or infection. ${ }^{3,7,8}$ In these two situations, the factors involved in etiopathogenesis are related to prematurity.

The critical period of cerebral myelination is between 23 and 32 weeks' postconceptional age, when pre-oligodendrocytes differentiate into immature oligodendrocytes, responsible for initiating myelination of the white matter. ${ }^{9}$

The reduction of cerebral perfusion in very small preterm neonates with deficient cerebrovascular autoregulation is a high risk for the occurrence of injuries to the periventricular white matter. ${ }^{8}$ This being so, preterm newborn infants exposed to intrauterine infections are vulnerable to pre-oligodendrocyte death in the face of ischemic insults, even when mild and insufficient to cause injuries by themselves. ${ }^{2,8-10}$

Many experimental and clinical studies have demonstrated the link between intrauterine infection and increased occurrence of PVL. ${ }^{11-13}$ A meta-analysis of 26 studies, relating chorioamnionitis to PVL, concluded that, among the newborn infants of mothers with chorioamnionitis, the chances of developing cystic PVL were three times greater. ${ }^{14}$ Notwithstanding, studies relating the presence of neonatal sepsis in extreme preterms with increased occurrence of PVL are rare and do not deal with this high risk population specifically.

The objective of our study was to investigate the presence of PVL and the possible risk factors associated with its development, particularly neonatal sepsis, in a population that is especially vulnerable to these conditions: very low birth weight preterm newborn infants (VLBWI).

\section{Methods}

This was a prospective cohort study of a population of newborn infants with birth weights between 500 and 1,500 g, born at the obstetrics center at our hospital and admitted to the neonatal intensive care unit (NICU) during the period between 1st August, 2005 and 31st July, 2007. Inclusion was based on the assessment of the duty physician in whose opinion the infants enrolled exhibited clinical signs suggestive of infection during the first week of life.

Exclusion criteria were death before 14 days of life, presence of major congenital malformations or malformation of the central nervous system, congenital STORCH infections (syphilis, toxoplasmosis, rubella, cytomegalovirus or herpes) or HIV+ mother.

An Informed Consent Form was read and signed by parents or guardians before the first cerebral ultrasound scan. The study was approved by Research Ethics Committee at the institution, protocol no. 04/446.
All newborn infants enrolled underwent a first cerebral ultrasound at 3 days of life; and then weekly until the sixth week of life or hospital discharge, if this took place before 6 weeks of life. This screening made it possible to diagnose PVL. Observational clinical monitoring while in NICU allowed for assessment of possible risk factors for PVL. VLBWI were discharged from hospital when they reached a weight of 2,000 $\mathrm{g}$, were able to receive full oral enteral feeding and were clinically stable.

After the serial ultrasound brain scans, patients were divided in two groups:

Group 1: VLBWI with diagnosis of PVL based on diffuse or cerebral cystic white matter injury.

Group 2: VLBWI without diagnosis of PVL, with cerebral echography negative for this condition.

Images were acquired via the anterior fontanelle, with a LOGIQ 500 Scanner (G. E. Medical Systems, United States) and an $11 \mathrm{MHZ}$ transducer. The researchers who carried out the scans were unaware of the patients' clinical status in terms of presence or absence of sepsis; images were recorded on the machine and reviewed by two researchers who were qualified in cerebral ultrasound scanning.

The criteria for diagnosing PVL were the presence of diffuse periventricular hyperechogenecity that persisted for a period of more than 7 days, without forming cysts (diffuse periventricular white matter injuries) or the presence of cystic lesions of at least $0.5 \mathrm{~cm}$ in diameter, distributed bilaterally and located close to the external angles of the lateral ventricles. ${ }^{6,15}$

The presence of ventricular dilatation without cerebral hemorrhage during any of the serial ultrasound scans was considered to be secondary to necrosis of the white matter present in the diffuse PVL component. ${ }^{3}$

The following data were collected prospectively and later compared between the two groups: sex, birth weight, gestational age, fifth minute Apgar score, SNAPPE-II score, antenatal corticosteroid use, neonatal sepsis, respiratory distress syndrome (RDS), peri-intraventricular hemorrhage (PIVH), non-hemorrhagic periventricular dilatation and pressure positive mechanical ventilation for more than 24 hours. Mortality was also compared between groups.

Gestational age was determined according to the mother's obstetric history (date of last menstruation) and confirmed by obstetric ultrasound, at 12 weeks' pregnancy at the latest. In the absence of any reliable maternal data, gestational age was determined by somatic physical and neurological examination of the newborn, using the New Ballard method. ${ }^{16}$

The SNAPPE-II score is a scale to indicate illness severity and risk of mortality during the neonatal period. ${ }^{17}$ SNAPPE-II scores were calculated for all infants within 12 hours of birth. 
Infants were diagnosed with RDS if they exhibited expiratory grunting, nostril flaring, sternum retraction, a need for $\geq$ $40 \%$ oxygen and a chest $X$ ray with diffuse ground-glass appearance and a need for exogenous surfactant.

Presence or absence of PIVH was determined from the first ultrasound brain scan and during weekly monitoring scans, according to the classification defined by Papile et al. ${ }^{18}$

The criteria for diagnosing neonatal sepsis were clinical signs of infection in the presence of microorganism in blood or cerebrospinal fluid cultures. Serum samples for blood culture were obtained using the aseptic collection technique and according to the recommendations laid out by Schelonka et al. ${ }^{19}$

Clinical signs of infection were the presence of at least three of the findings referred to below, or two clinical findings plus one or more maternal risk factors ${ }^{20,21}$ : thermal instability; apnea, bradypnea, grunting, tachypnea, sternum and subcostal retraction, nostril flaring and cyanosis; hypotonia and convulsions; irritability and lethargy; gastrointestinal symptoms, such as abdominal distension, vomiting, gastric residues and difficulty accepting food; idiopathic jaundice; cutaneous pallor, cold skin, perspiration, hypotension and capillary refill time longer than $3 \mathrm{~s}$; signs of bleeding, with clinical status suggestive of disseminated intravascular coagulation; subjective assessment: the newborn "does not look well".

Maternal risk factors were obtained from their obstetric records and their medical and perinatal histories. The events most often described were maternal fever, urinary infection and more than 18 hours of rupture of membranes.

The sample size was calculated from published data reporting the occurrence of $\mathrm{PVL}$ at 7 to $26 \%$ of infants born preterm and weighing less than $1,500 \mathrm{~g}$ using proportions. Based on a statistical power of $90 \%$ with a level of significance of $5 \%(a=0.05)$ and an estimated PVL incidence of $33 \%$ in high risk populations (VLBWI with presumed or confirmed sepsis), the resulting sample size was 30 newborn infants in each group. ${ }^{10}$

\section{Statistical analysis}

Data are presented in the form of mean and standard deviation or median and interquartile range (from the 25 th to the 75th percentiles), depending on the behavior of the variable in question. Differences between means were analyzed using Student's $t$ test, and differences between medians were subject to the Mann-Whitney test. Categorical variables were analyzed using the chi-square test. Variables were included in the logistic regression model is they exhibited statistical significance $(p<0.05)$ in the comparison between groups, and their odds ratios as risk factors for PVL were calculated. The level of significance was $p<0.05$.

\section{Results}

A total of 180 VLBWI were born during the 2 years of this study, 88 of whom met the inclusion criteria and were followed up until their sixth week of life, hospital discharge or later death (after 14 days of life). A total of 26 VLBWI (29.5\%) died while in NICU, and 62 (70.5\%) were discharged from hospital. Nasal Continuous Positive Airway Pressure (CPAP) was used in 85 (96.6\%) newborn infants, since it is routine at our service to install nasal CPAP for all VLBWI as soon as they exhibit any type of respiratory distress. Antenatal corticosteroids were given to 51 patients ( $58 \%$ of the population) and nine of these had PIVH, in contrast with 25 out of 37 newborn infants whose mothers had not been given antenatal corticosteroids $(p<0.001)$.

The exposure group (group 1) comprised 51 VLBWI with $P V L$, and was compared with 37 VLBWI in the group without PVL (group 2). Type of delivery, presence of perinatal asphyxia, necrotizing enterocolitis and persistent ductus arteriosus were similar between groups. With the exception of five newborn infants who exhibited ventricular dilatation which could not be defined as either secondary to PVL or post-hemorrhagic, ventricular dilatation was significantly more frequent among VLBWI with PVL than among those without PVL. Three newborn infants without PVL exhibited ventricular dilatation secondary to cerebral hemorrhage diagnosed by means of serial ultrasound scans (Table 1 ). Neonatal sepsis and the use of mechanical ventilation with intermittent positive pressure were significantly more frequent among newborn infants with PVL (Table 1).

The logistic regression model employed took the presence of PVL as dependent factor, and as independent factors just those variables that had exhibited statistical significance in the univariate analyses $(p<0.05)$ and were not secondary to PVL. Therefore, just neonatal sepsis and mechanical ventilation were analyzed as independent factors, and both proved significantly associated with PVL (Table 2 ).

Twelve newborn infants with PVL had positive blood cultures: eight Staphylococcus coagulase negative, two Streptococcus agalactiae, one Streptococcus a-hemolitico and one Escherichia coli. Just one newborn without PVL had a blood culture positive for Staphylococcus coagulase negative. Staphylococcus coagulase negative was present in $69.2 \%$ of cases of sepsis and was the most prevalent germ.

\section{Discussion}

In our 2 year cohort, more VLBWI with PVL had sepsis than newborn infants without PVL and more of them required intermittent positive pressure mechanical ventilation for more than 24 hours, despite there being no significant difference in incidence of RDS or degree of immaturity. The mean gestational ages of the two groups were the same ( 29 weeks). Those findings suggest that a greater need for invasive ventilatory support and presence of sepsis were more related to PVL than prematurity in this population. 
Table 1 - Periventricular leukomalacia in very low birth weight infants and possible risk factors

\begin{tabular}{|c|c|c|c|}
\hline VLBWI $(n=88)$ & PVL present $(n=51)$ & PVL absent $(n=37)$ & $\mathbf{p}$ \\
\hline Males (\%) & $32(62.7 \%)$ & $25(67.5 \%)$ & 0.44 \\
\hline Birth weight $(\mathrm{g})^{*}$ & $1,010 \pm 250$ & $1,068 \pm 270$ & 0.29 \\
\hline Gestational age (weeks)* & $29.2 \pm 2.5$ & $29.5 \pm 2.3$ & 0.15 \\
\hline \multicolumn{4}{|l|}{ Type of delivery } \\
\hline Vaginal & $17(33.3 \%)$ & $11(29.7 \%)$ & 0.63 \\
\hline Caesarian & $34(66.7 \%)$ & $26(70.3 \%)$ & 0.63 \\
\hline Antenatal corticosteroids & $29(57 \%)$ & $22(60 \%)$ & 0.53 \\
\hline 5-minute Apgar score & $8(6-9)$ & $8(7-9)$ & 0.12 \\
\hline SNAPPE-II ${ }^{+}$ & $18(7-29)$ & $17(0-30)$ & 0.097 \\
\hline $\begin{array}{l}\text { Peri-intraventricular } \\
\text { hemorrhage (degrees } \\
\text { I+II+III+IV) }\end{array}$ & $21(41 \%)$ & $13(35.1 \%)$ & 0.25 \\
\hline Ventricular dilatation $(n=15)$ & $12 / 15(80 \%)$ & $3 / 15(20 \%)$ & 0.046 \\
\hline Neonatal sepsis & $12(23.5 \%)$ & $1(2.7 \%)$ & 0.005 \\
\hline Respiratory distress syndrome & $32(62.7 \%)$ & $21(56.7 \%)$ & 0.12 \\
\hline $\begin{array}{l}\text { Mechanical ventilation > } 24 \\
\text { hours }\end{array}$ & $44(86 \%)$ & $22(59 \%)$ & 0.004 \\
\hline Late neonatal mortality & $15(29.4 \%)$ & $11(29.7 \%)$ & 0.45 \\
\hline
\end{tabular}

$\mathrm{PVL}=$ periventricular leukomalacia; VLBWI = very low birth weight infants.

Data presented as mean \pm standard deviation, median (interquartile range), $\mathrm{n}(\%)$, chi-square test or Fisher's exact test when not indicated otherwise.

* Student's $t$ test.

+ Mann-Whitney test.

Table 2 - Neonatal sepsis and mechanical ventilation as risk factors for periventricular leukomalacia in very low birth weight infants (logistic regression)

\begin{tabular}{lccc}
\hline & OR & CI & P \\
\hline Confirmed sepsis & 11.613 & $(1.42-94.9)$ & 0.027 \\
Mechanical ventilation & 4.3 & $(1.32-14.43)$ & 0.015 \\
\hline
\end{tabular}

$\mathrm{CI}=$ confidence interval; $\mathrm{OR}=$ odds ratio.

Shalak \& Perlman have written about the importance of antenatal corticosteroid therapy for reducing severe cerebral hemorrhage, since it accelerates maturation of the germinal matrix, increases systemic arterial pressure with improved cerebral perfusion and appears to be associated with less severe cases of RDS. ${ }^{22}$ Our data demonstrate that more than half of our VLBWI ( $58 \%$ ) had been given antenatal corticosteroid, and that $66 \%$ of those did not present hemorrhage or exhibit level I PIVH with later reabsorption, demonstrating the efficacy of this preventative measure for reducing the occurrence of cerebral hemorrhage inpremature newborn infants, since those who were given antenatal corticosteroid suffered significantly fewer cerebral hemorrhages.

It has been suggested that antenatal corticosteroid may be protective against injuries to cerebral white matter in
VLBWI due to its anti-inflammatory function. ${ }^{23}$ In our study, a complete course of antenatal corticosteroid was given to $57 \%$ of the mothers whose newborn infants developed PVL and to $60 \%$ of the mothers of newborn infants without PVL; its administration did not result in a reduced incidence of PVL among VLBWI.

Neonatal sepsis is a clinical condition that activates a cascade of acute inflammatory response mediators. Kadhim et al., in two of their studies, found evidence of significant expression of tumor necrosis factor alpha (TNF-a) and, to a lesser extent, of interleukin-1 beta (IL-1 $\beta$ ) in cerebral tissue with PVL, with increased cytokines production in cases where $P V L$ was associated with infection. The inflammatory reaction was detected at an early stage of PVL and extended up to the last phase, cavitation. 24,25 
Prematurity is a considerable neonatal risk factor for PVL, especially for white matter injuries with a diffuse component (non-cystic). There are speculations that other neonatal conditions are associated with diagnosis of $\mathrm{PVL}$, such as perinatal asphyxia, hypovolemia and early neonatal sepsis. Many of these factors cause a reduction in systemic blood pressure, although clinical studies have not demonstrated an association between PVL and postnatal systemic hypotension. 22,26,27 It was recently suggested that alterations in cerebral blood flow are a necessary condition for PVL, but are not enough to cause it in isolation. The preference for premature infants is related to the timing of the insult and the distribution of susceptible pre-oligodendrocytes around the brain. ${ }^{8} \mathrm{~A}$ question that remains is the influence of the cascade of inflammatory events that occurs in neonatal sepsis. The concern of this study, however, was to exclusively select a population at greater risk of suffering PVL, i.e. sick VLBWI, although not necessarily with a definite diagnosis of neonatal sepsis.

The presence of ischemia, inflammation or systemic infection appears to be etiopathogenic for PVL. It is possible that PVL in preterm newborns is associated with an altered inflammatory response equilibrium within the central nervous system, and that proinflammatory cytokines act directly on the brain, and not as part of a larger systemic response. ${ }^{10}$ Studies that have assessed the association between maternal chorioamnionitis and PVL have not examined the occurrence of sepsis in premature newborns. ${ }^{11-14}$ Our study assessed neonatal sepsis as an important risk factor for PVL in VLBWI. We did not asses the presence of chorioamnionitis because we did not have histology for all placentas and, consequently, lacked diagnoses of subclinical chorioamnionitis, which is a risk factor for PVL that has already been described. ${ }^{28}$

We avoided possible sample selection bias, defining neonatal sepsis as present when newborn infants had a positive culture. For this reason we can safely say that the occurrence of neonatal sepsis in VLBWI increases their chances of PVL by 11 times. In the majority of cases, PVL has a subclinical presentation, and it is therefore of fundamental importance to understand its risk factors, which, together with serial ultrasound brain scans, make an early diagnosis possible. It is possible to detect PVL by means of transfontanelar cerebral ultrasound, as long as the newborn infant is monitored weekly and up to hospital discharge. This is also useful to predict the development of cerebral palsy. ${ }^{6,29-31}$ Vollmer et al. studied preterm newborns at 24 to 32 weeks' gestational age and assessed the efficacy of cerebral ultrasound for detection of cerebral lesions and their relation with delayed neuropsychomotor development at 8 years of age. These authors found that poor prognosis depended fundamentally on the presence and type of lesion found with cerebral ultrasound. ${ }^{26}$ It is possible that serial cerebral ultrasound scans, in addition to clinical findings from VLBWI in neonatal intensive care units, such as neonatal sepsis, are important prognostic factors.
One possible limitation of our study was that we did not use magnetic resonance imaging to confirm the diagnosis of $P V L$, especially the diffuse component of white matter injuries. Although magnetic resonance imaging has demonstrated greater diagnostic sensitivity and specificity for PVL, the elevated costs and technical difficulties involved, such as the need for transportation from NICU to radiology department, limits its use as a routine, ${ }^{7,30}$ whereas cerebral ultrasound scans are accessible, practical and well-adapted to bedside use. ${ }^{15}$ We therefore believe that our study makes an additional contribution by demonstrating that it is possible to reach a diagnosis of PVL using ultrasound and following an appropriate follow-up protocol.

We already know that maternal chorioamnionitis is a risk factor for PVL. In this study we demonstrated that neonatal sepsis is also an important risk factor for PVL in VLBWI with clinical suspicion of neonatal infection. It is probable that the systemic inflammatory response to perinatal and neonatal infections is the principal factor involved in the etiopathogenesis of PVL, which is a disease with significant morbidity, in common with cerebral palsy. Further studies attempting to block this inflammatory response may be promising for reducing the magnitude of the PVL and, as a consequence, improve the future quality of life of these VLBWI.

\section{Acknowledgements}

The authors would like to thank Vânia Naomi Hirakata and Daniela Benzano, for their help with the statistical analysis.

\section{References}

1. Vohr B, Wright LL, Dusick AM, Mele L, Verter J, Steichen JJ, et al. Neurodevelopmental and functional outcomes of extremely low birth weight infants in the National Institute of Child Health and Human Development Neonatal Research Network, 1993-1994. Pediatrics. 2000;105:1216-26.

2. Wood NS, Marlow N, Costeloe K, Gibson AT, Wilkinson AR. Neurologic and developmental disability after extremely preterm birth. EPICure Study Group. N Engl J Med. 2000;343:378-4.

3. Volpe JJ. Cerebral White matter injury of the preterm infant-more common than you think (commentaries). Pediatrics. 2003;112: 176-80.

4. Msall ME. Neurodevelopmental surveillance in the first 2 years after extremely preterm birth:evidence, challenges, and guidelines. Early Hum Dev. 2006;82:157-66.

5. Pagliano E, Fedrizzi E, Erbetta A, Bulgheroni S, Solari A, Bono R. Cognitive profiles and visuoperceptual abilities in preterm and term spastic diplegic children with periventricular leukomalacia. J Child Neurol. 2007;22:282-8.

6. Leijser LM, de Vries LS, Cowan FM. Using cerebral ultrasound effectively in the newborn infant. Early Hum Dev. 2006;82: 827-35.

7. Maalouf EF, Duggan PJ, Counsell SJ, Rutherford MA, Cowan F, Azzopardi $D$, et al. Comparison of findings on cranial ultrasound and magnetic resonance imaging in preterm infants. Pediatrics. 2001;107:719-27. 
8. Back SA, Riddle A, McClure MM. Maturation-dependent vulnerability of perinatal white matter in premature birth. Stroke. 2007;38:724-30.

9. Back SA, Luo NL, Borenstein NS, Volpe JJ, Kinney HC. Arrested oligodendrocyte lineage progression during human cerebral white matter development: dissociation between the timing of progenitor differentiation and myelinogenesis. J Neuropathol Exper Neurol. 2002;61:197-211.

10. Ellison VJ, Mocatta TJ, Winterbourn CC, Darlow BA, Volpe JJ, Inder TE. The relationship of CSF and plasma cytokine levels to cerebral white matter injury in the premature newborn. Pediatr Res. 2005; 57:282-6.

11. Yoon BH, Romero R, Park JS, Kim M, Oh SY, Kim CJ, et al. The relationship among inflammatory lesions of the umbilical cord (funisitis), umbilical cord plasma interleukin-6 concentration, amniotic fluid infection, and neonatal sepsis. Am J Obstet Gynecol. 2000;183:1124-9.

12. Dammann O, Phillips TM, Allred EN, O'Shea TM, Paneth N, Van Marter LJ, et al.; Elgan Study Investigators. Mediators of fetal inflammation in extremely low gestational age newborns. Cytokine. 2001;13:234-9.

13. Baud O, Emilie D, Pelletier E, Lacaze-Masmonteil T, Zupan V, Fernandez $\mathrm{H}$, et al. Amniotic fluid concentrations of interleukin-1 $\beta$, interleukin- 6 and TNF-a in chorioamnionitis before 32 weeks of gestation: histological associations and neonatal outcome. Br J Obstet Gynaecol. 1999;106:72-7.

14. Wu YW, Colford JM Jr. Chorioamnionitis as a risk factor for cerebral palsy (a meta-analysis). JAMA. 2000;284:1417-24.

15. Leijser LM, de Vries LS, Rutherford MA, Manzur AY, Groenendaal F, de Koning TJ, et al. Cranial ultrasound in metabolic disorders presenting in the neonatal period: characteristic features and comparison with MR Imaging. AJNR Am J Neuroradiol. 2007;28:1223-31.

16. Ballard JL, Khoury JC, Wedig K, Wang L, Eilers-Walsman BL, Lipp R. New Ballard Score, expanded to include extremely premature infants. J Pediatr. 1991;119:417-23.

17. Richardson DK, Corcoran JD, Escobar GJ, Lee SK. SNAP-II and SNAPPE-II: Simplified newborn illness severity and mortality risk scores. J Pediatr. 2001;138:92-100.

18. Papile L, Burstein J, Burstein R, Koffler $H$. Incidence and evaluation of subependymal haemorrhage: a study of children with a birthweight less than 1,500 gm. J Pediatr. 1978;92: 529-34.

19. Schelonka RL, Chai MK, Yoder BA, Hensley D, Brockett RM, Ascher DP. Volume of blood required to detect common neonatal pathogens. J Pediatr. 1996;129:275-8.
20. Buck C, Bundschu J, Gallati H, Bartmann P, Pohlandt F. Interleukin-6: a sensitive parameter for the early diagnosis of neonatal bacterial infection. Pediatrics. 1994;93:54-8.

21. de Bont ES, Martens A, Van Raan J, Samson G, Fetter WP, Okken A, et al. Tumor necrosis factor-alpha, interleukin-1 beta, and interleukin-6 plasma levels in neonatal sepsis. Pediatr Res. 1993;33:380-3.

22. Shalak L, Perlman JM. Hemorrhagic-ischemic cerebral injury in the preterm infant: current concepts. Clin Perinatol. 2002;29: 745-63.

23. Agarwal R, Chiswick ML, Rimmer S, Taylor GM, McNally RJ, Alston $\mathrm{RD}$, et al. Antenatal steroids are associated with a reduction in the incidence of cerebral white matter lesions in very low birthweight infants. Arch Dis Child Fetal Neonatal Ed. 2002;86: F96-F101.

24. Kadhim H, Tabarki B, Verellen G, De Prez C, Rona AM, Sébire G. Inflammatory cytokines in the pathogenesis of periventricular leukomalacia. Neurology. 2001;56:1278-84.

25. Kadhim H, Tabarki B, de Prez C, Rona AM, Sébire G. Interleukin-2 in the pathogenesis of perinatal white matter damage. Neurology. 2002;58:1125-8.

26. Vollmer B, Roth S, Baudin J, Stewart AL, Neville BG, Wyatt JS. Predictors of long-term outcome in very preterm infants: gestational age versus neonatal cranial ultrasound. Pediatrics. 2003;112:1108-14.

27. Volpe J]. Neurobiology of periventricular leukomalacia in the premature infant. Pediatr Res. 2001;50:553-62.

28. Perlman JM, Risser R, Broyles S. Bilateral cystic periventricular leukomalacia in the premature infant: associated risk factors. Pediatrics. 1996;97:822-7.

29. De Vries LS, van Haastert IC, Rademaker KJ, Koopman C, Groenendaal F. Ultrasound abnormalities preceding cerebral palsy in high-risk preterm infants. J Pediatr. 2004;144:815-20.

30. Inder TE, Anderson NJ, Spencer C, Wells S, Volpe JJ. White matter injury in the premature infant: a comparison between serial cranial sonographic and MR findings at term. AJNR Am J Neuroradiol. 2003;24:805-9.

31. Silveira RC, Procianoy RS. Lesões isquêmicas cerebrais no recém-nascido pré-termo de muito baixo peso. J Pediatr (Rio J). 2005;81:S23-32.

Correspondence:

Renato S. Procianoy

Rua Silva Jardim, 1155/701

CEP 90450-071 - Porto Alegre, RS - Brazil

Tel.: + 55 (51) 3331.5726

Fax: +55 (51) 3331.2738

E-mail: renatosp@terra.com.br 